ESAIM: PROCEEDINGS, October 2011, Vol. 32, p. 95-102

E. Cancès, N. Crouseilles, H. Guillard, B. Nkonga, and E. Sonnendrücker, Editors

\title{
DIRECTIONAL AND SCALE-DEPENDENT STATISTICS OF QUASI-STATIC MAGNETOHYDRODYNAMIC TURBULENCE
}

\author{
Naoya Okamoto ${ }^{1},{\text { Katsunori } \text { Yoshimatsu }^{2}, \text { Kai Schneider }^{3} \text { and Marie Farge }}^{4}$
}

\begin{abstract}
Anisotropy and intermittency of quasi-static magnetohydrodynamic (MHD) turbulence in an imposed magnetic field are examined, using three-dimensional orthonormal wavelet analysis. Wavelets are an efficient tool to examine directional scale-dependent statistics, since they are based on well-localized functions in space, scale and direction. The analysis is applied to two turbulent MHD flows computed by direct numerical simulation with $512^{3}$ grid points and with different intensities of the imposed magnetic field. It is found that the imposed magnetic field plays an important role on the amplification of anisotropy and intermittency of the flow.

Résumé. L'anisotropie et l'intermittence de la turbulence magnétohydrodynamique (MHD) dans l'approximation quasi-statiqueavec un champ magnétique imposé sont examinés en utilisant l'analyse par ondelettes orthogonales. Les ondelettes sont un outil efficace pour examiner les statistiques directionnelles en fonction de l'échelle, car elles sont basées sur des fonctions bien localisées dans l'espace, l'échelle et l'orientation. L'analyse est appliquée à deux écoulements MHD turbulents calculés par simulation numérique directe avec $512^{3}$ points de la grille à différentes intensités du champ magnétique imposé. Il se trouve que le champ magnétique imposé joue un rôle important sur l'amplification de l'anisotropie et de l'intermittence de l'écoulement.
\end{abstract}

\section{INTRODUCTION}

Low-magnetic-Reynolds-number magnetohydrodynamic (MHD) turbulence with an imposed magnetic field is ubiquitous in industrial applications, such as electro-magnetic processing of materials in metallurgical industry [1]. In this context the so-called quasi-static approximation can be applied to MHD turbulence (see e.g. [2]).

Characteristic features of quasi-static MHD turbulence subject to an imposed magnetic field are multiscale anisotropy and intermittency of the flow. Quasi-static MHD turbulence has been studied by means of direct numerical simulation (DNS) and different tools to quantify the anisotropy have been developed so far [3]. Using energy spectra in Fourier space, it is shown that the anisotropy is persistent at small scale [4-6]. These spectral analyses, while capturing the multiscale character, cannot quantify the spatially intermittent nature of the flows. However, the wavelet representation can simultaneously examine the multiscale distribution of a quantity, its anisotropy, and in addition yield information on its spatial distribution, since the representation is based on well-localized functions in space, scale and direction [7]. The multiscale dynamics of both hydrodynamic and MHD turbulence have motivated the development of wavelet techniques to analyze [8-12], model [13,14] and also

\footnotetext{
${ }^{1}$ Center for Computational Science, Nagoya University, Nagoya, 464-8603, Japan (e-mail: okamoto@ccs.engg.nagoya-u.ac.jp)

2 Department of Computational Science and Engineering, Nagoya University, Nagoya, 464-8603, Japan

3 M2P2-CNRS \& CMI Aix-Marseille University, 39 rue F. Joliot-Curie, 13453 Marseille Cedex 13, France

4 LMD-IPSL-CNRS, Ecole Normale Supérieure, 24 rue Lhomond, 75231 Paris Cedex 05, France
}

(C) EDP Sciences, SMAI 2011 
to compute such flows [15]. Bos et al. introduced directional scale-dependent statistics to measure intermittency of anisotropic turbulence [10].

The aim of this paper is to examine anisotropy and intermittency of the quasi-static MHD turbulence, by using directional and scale dependent statistics [10]. We study the influence of the strength of the magnetic field on the intermittency and the anisotropy. The statistics of two turbulent MHD flows with different intensities of the imposed magnetic field are compared with those of an isotropic random field.

The remainder of the paper is organized as follows. First we briefly describe the DNS of the quasi-static MHD turbulence in section 2. The directional scale-dependent statistics based on orthonormal wavelet decomposition are introduced in section 3. Section 4 presents numerical results and a conclusion is drawn in section 5 .

\section{Direct Numerical Simulation of quasi-Static MHD turbulence}

We consider homogeneous MHD turbulence of incompressible fluid in a uniform magnetic field $\boldsymbol{B}_{0}$ in a $2 \pi$ periodic box. In the limit of low magnetic Reynolds number, the motion of the flow obeys the following equations under the so-called quasi-static MHD approximation [2],

$$
\begin{aligned}
\frac{\partial}{\partial t} \boldsymbol{u}+(\boldsymbol{u} \cdot \nabla) \boldsymbol{u} & =-\frac{1}{\rho} \nabla p+\nu \Delta \boldsymbol{u}-\frac{\sigma}{\rho} \Delta^{-1}\left(\boldsymbol{B}_{0} \cdot \nabla\right)^{2} \boldsymbol{u}+\boldsymbol{f} \\
\nabla \cdot \boldsymbol{u} & =0 .
\end{aligned}
$$

Here, $\boldsymbol{u}$ is the velocity field, $\rho$ the fluid density, $\nu$ the kinematic viscosity and $\sigma$ the electrical conductivity. The constant magnetic field is given by $\boldsymbol{B}_{0}=\left(0,0, B_{0}\right)$. The third term in right-hand side of the equation (1) is the rotational part of the Lorentz force, and the modified pressure $p$ includes both the fluid pressure and the magnetic pressure. The symbol $\Delta^{-1}$ represents the inverse Laplace operator. An estimate of the ratio of the Lorentz force to the nonlinear term in (1) is given by the interaction parameter $N=\sigma B_{0}^{2} L /\left(\rho u^{\prime}\right)$, where $u^{\prime}=\left\langle\boldsymbol{u}^{2}\right\rangle / 3$ and $L$ is the integral length scale defined by $L=\pi /\left(2 u^{\prime 2}\right) \int E(k) / k d k$. Here, $\langle\cdot\rangle$ denotes the spatial average and $E(k)$ is the energy spectrum.

We performed DNS computations at two interaction parameters $N=1$ and 3 . We used a Fourier pseudospectral method with a fourth-order Runge-Kutta method for time marching. The aliasing errors are removed by a phase-shift method, which keeps all the Fourier modes satisfying $k=|\boldsymbol{k}|<k_{\max }$, where $\boldsymbol{k}$ is the wave vector, $k_{\max }=2^{1 / 2} n^{1 / 3} / 3$ and $n$ the number of the grid points. An external solenoidal random force $\boldsymbol{f}$ is applied to the velocity field only in the low wavenumber range, $k<2.5$. Readers interested in details of how to generate such a random force are referred to the Appendix of [16].

The initial conditions and the flow parameters, except the interaction parameter, are identical in the two simulations. The grid points are $n=512^{3}$, the kinematic viscosity $\nu=2.8 \times 10^{-4}$ and the time increment $\Delta t=1.0 \times 10^{-3}$. The two computations are integrated during about nine initial eddy turnover times $T=L / u^{\prime}$. The Kolmogorov length scale $\eta$ is defined by $\eta=\left(\nu^{3} /\langle\epsilon\rangle\right)^{1 / 4}$, where $\langle\epsilon\rangle$ is the mean rate of energy dissipation per unit mass. Table 1 summarizes the Taylor microscale Reynolds number $R_{\lambda}=u^{\prime} \lambda / \nu$, where $\lambda=\left(15 \nu u^{\prime 2} /\langle\epsilon\rangle\right)^{1 / 2}$, $k_{\max } \eta$ at $t=9 T$ and the interaction parameter $N$. The initial energy spectrum of the DNS has the form $E(k) \propto k^{4} \exp \left(-2 k^{2} / k_{p}^{2}\right)$, where $k_{p}=2$ and the initial total energy is 0.5 .

\begin{tabular}{ccc}
$N$ & $R_{\lambda}$ & $k_{\max } \eta$ \\
\hline 1 & 390 & 1.4 \\
3 & 927 & 2.0
\end{tabular}

TABLE 1. Physical parameters of the DNS computations with $N=1$ and 3 at $t=9 T$. 


\section{Methodology}

To examine anisotropy and intermittency of MHD turbulence, we first introduce the directional statistical quantities based on the orthogonal wavelet representation. Then we compare the statistics of the DNS field thus obtained with those of an isotropic divergence free random field.

\subsection{Directional statistical quantities based on the wavelet representation}

The velocity field $\boldsymbol{u}=\left(u^{1}, u^{2}, u^{3}\right)$, sampled at resolution $n=2^{3 J}$, is decomposed into an orthogonal wavelet series such that

$$
\boldsymbol{u}(\boldsymbol{x})=\sum_{\lambda} \widetilde{\boldsymbol{u}}_{\lambda} \psi_{\lambda}(\boldsymbol{x})
$$

where the multi-index $\lambda=(j, \boldsymbol{i}, d)$ denotes, for each wavelet $\psi_{\lambda}$, the scale index $j$ (varying from 0 to $J-1$ ), the spatial index $\boldsymbol{i}=\left(i_{1}, i_{2}, i_{3}\right)$, having $2^{3 j}$ values for each $j$ and $d$, and the direction index $d=1, \ldots, 7$ [8,9]. Here, we use Coiflet 12 wavelets [9]. Due to orthogonality, the wavelet coefficients are given by $\widetilde{\boldsymbol{u}}_{\lambda}=\left\langle\boldsymbol{u}, \psi_{\lambda}\right\rangle$, where $\langle\cdot, \cdot\rangle$ denotes the $L^{2}$-inner product. The wavelet coefficients measure the fluctuations of $\boldsymbol{u}$ at scale $2^{-j}$ and around position $\boldsymbol{i} / 2^{j}$ for each of the seven directions $d$. The contribution of $\boldsymbol{u}$ at scale $2^{-j}$ and direction $d$, denoted by $\boldsymbol{u}_{j, d}$, is obtained by fixing $(j, d)$ and summing only over $\boldsymbol{i}$ in Eq. (3). By construction we have $\boldsymbol{u}(\boldsymbol{x})=\sum_{j=0}^{J-1} \sum_{d=1}^{7} \boldsymbol{u}_{j, d}(\boldsymbol{x})$.

Relating scale $2^{-j}$ with wavenumber $k_{j}$ as $k_{j}=k_{\psi} 2^{j}$, where $k_{\psi}$ is the centroid wavenumber of the chosen wavelet $\left(k_{\psi}=0.77\right.$ for the Coiflet 12 used here), we obtain the directional wavelet energy spectrum for the velocity component $u^{\ell}$, defined by

$$
\widetilde{E}^{\ell}\left(k_{j}, d\right)=\left\langle e_{j, d}^{\ell}\right\rangle / \Delta k_{j}
$$

where $e_{j, d}^{\ell}=\left(u_{j, d}^{\ell}\right)^{2} / 2$ and $\Delta k_{j}=\left(k_{j+1}-k_{j}\right) \ln 2$. Its standard deviation is given by

$$
\tilde{\sigma}^{\ell}\left(k_{j}, d\right)=\sqrt{\left\langle\left(e_{j, d}^{\ell}\right)^{2}\right\rangle-\left\langle e_{j, d}^{\ell}\right\rangle^{2}} / \Delta k_{j}
$$

From the directional scale-dependent energy spectrum for all the velocity components, we obtain the total energy $E=\sum_{\ell, j, d} \widetilde{E}^{\ell}\left(k_{j}, d\right)$.

To study higher-order statistics, we define the flatness of $u^{\ell}$ at scale $2^{-j}$ and direction $d$ by

$$
F_{j, d}^{\ell}=\frac{\left\langle\left(u_{j, d}^{\ell}(\boldsymbol{x})\right)^{4}\right\rangle}{\left\langle\left(u_{j, d}^{\ell}(\boldsymbol{x})\right)^{2}\right\rangle^{2}} .
$$

In [10] it was shown that the flatness is related to the standard deviation of the spectral distribution of energy (5) by

$$
F_{j, d}^{\ell}=\left(\frac{\tilde{\sigma}^{\ell}\left(k_{j}, d\right)}{\widetilde{E}^{\ell}\left(k_{j}, d\right)}\right)^{2}+1 .
$$

This demonstrates that the scale-dependent flatness $F_{j, d}^{\ell}$ based on the wavelets coefficients of velocity yields an easy way to compute a quantitative measure of the spatial variability of the energy spectrum.

Summing the velocity field $\boldsymbol{u}_{j, d}(\boldsymbol{x})$ from $d=1$ to 7 yields the contribution of $\boldsymbol{u}$ at only scale $j$, i.e., $\boldsymbol{u}_{j}(\boldsymbol{x})=$ $\sum_{d} \boldsymbol{u}_{j, d}(\boldsymbol{x})$. Replacing $e_{j, d}^{\ell}$ in Eqs. (4) and (5) by $e_{j}^{\ell}=\left(u_{j}^{\ell}\right)^{2} / 2$, we obtain expressions for the wavelet energy spectrum and its standard deviation,

$$
\widetilde{E}^{\ell}\left(k_{j}\right)=\left\langle e_{j}^{\ell}\right\rangle / \Delta k_{j}, \quad \widetilde{\sigma}^{\ell}\left(k_{j}\right)=\sqrt{\left\langle\left(e_{j}^{\ell}\right)^{2}\right\rangle-\left\langle e_{j}^{\ell}\right\rangle^{2}} / \Delta k_{j}
$$

The wavelet spectrum $\widetilde{E}^{\ell}\left(k_{j}\right)$ corresponds to a smoothed version of the Fourier energy spectrum [8,9]. The flatness of $u_{j}$ is given by $F_{j}^{\ell}=\left\{\widetilde{\sigma}^{\ell}\left(k_{j}\right) / \widetilde{E}^{\ell}\left(k_{j}\right)\right\}^{2}+1$. 
In the present paper the considered flows are axisymmetric with respect to the $x_{3}$-axis. Hence, we consider for the directional statistics only three principle directions, i.e., $d=1,2$ and 3 .

\subsection{Divergence-free isotropic random field}

In addition to the two turbulent DNS fields we generated an isotropic divergence-free Gaussian random field $\boldsymbol{u}$ whose energy spectrum $E(k)$ is identical to the DNS field with $N=3$. This allows to compare the statistics of the DNS fields with that of the random field. A departure from the random field may provide a basic measure of the anisotropy and intermittency. We checked that the statistical behavior of the random field at $N=3$ are qualitatively the same as those for the random field at $N=1$.

\section{Numerical RESUlts}

\subsection{Visualization and wavelet energy spectra}

To obtain an intuitive idea on the flow structures at $N=1$ and 3 , we visualize the modulus of the vorticity field $|\boldsymbol{\omega}|$ in Figure 1. In Figure 1(left), one can see sheet-like structures and tube-like structures. Many of the structures are aligned parallel with the imposed magnetic field $\boldsymbol{B}_{0}$. The alignment of the structures shows the strong anisotropy of the flow. In contrast, the structures at $N=1$ in Figure 1 (right) exhibit entangled vortex tubes, which do not seem to be aligned with any specific direction. Thus, the field at $N=1$ is less anisotropic than that at $N=3$.

The anisotropy of the turbulent fields, i.e., the DNS fields, can be characterized by wavelet energy spectra for each component of the velocity fields $\boldsymbol{u}$. Figure 2 shows wavelet mean energy spectra $\widetilde{E}^{\ell}\left(k_{j}\right)$ for the DNS fields as a function of the dimensionless wavenumber $k_{j} \eta$. For the field with $N=3$, the spectrum of the
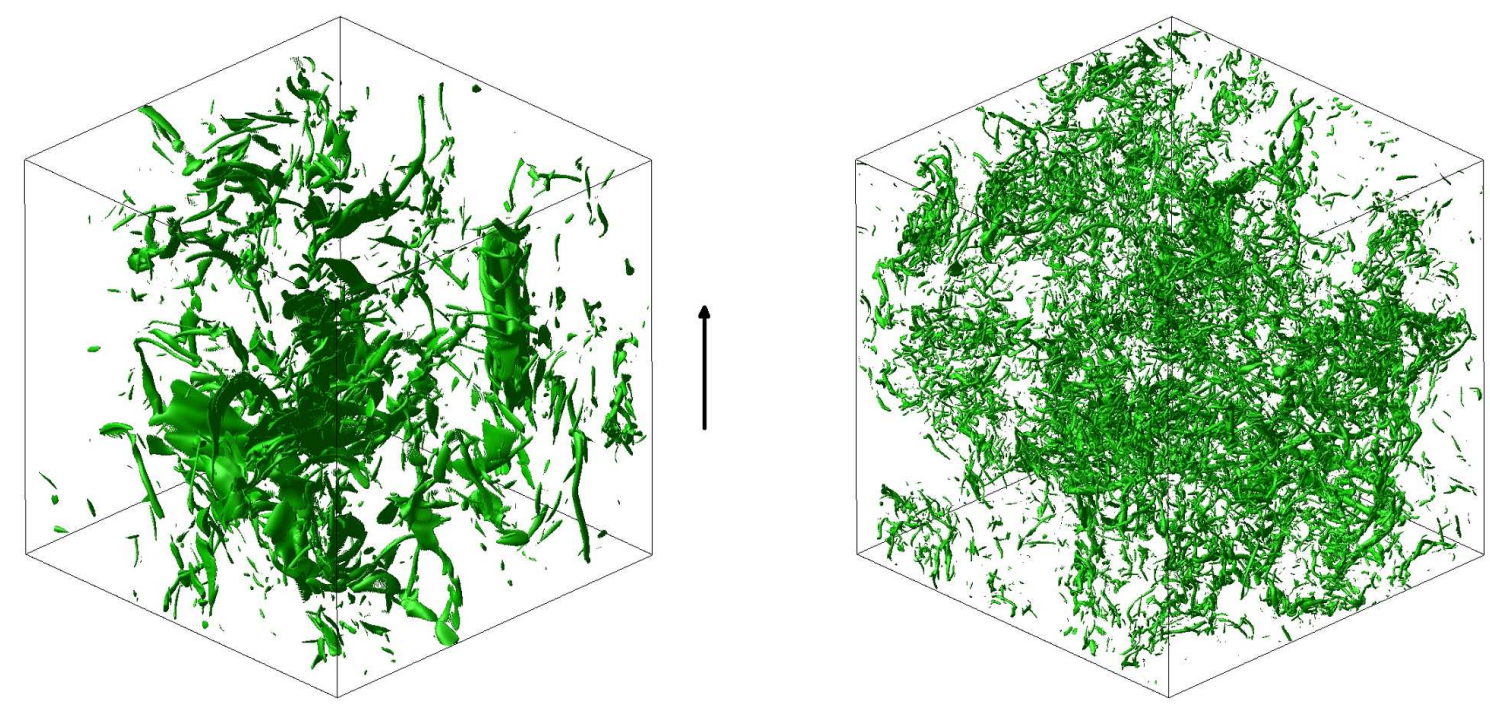

FiguRE 1. Visualization of intense vorticity regions of the DNS fields for $N=3$ (left) and $N=1$ (right) at $t=9 T$. Isosurfaces of $|\boldsymbol{\omega}|=\langle|\boldsymbol{\omega}|\rangle+4 \sigma$ are shown, where $\sigma$ denotes the standard deviation of $|\boldsymbol{\omega}|$. The vertical arrow indicates the direction of the imposed magnetic field. 

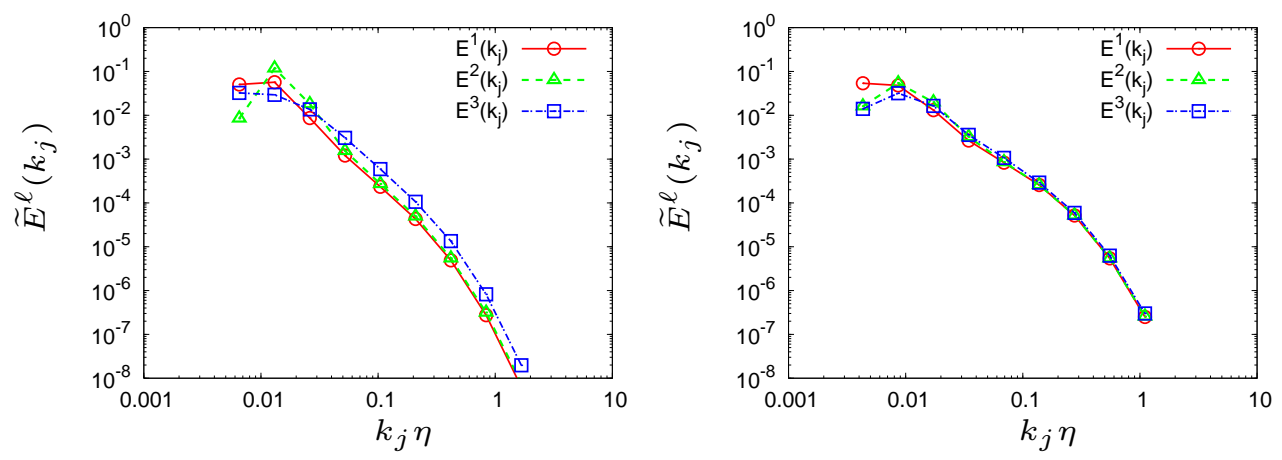

Figure 2. Wavelet mean energy spectra $\widetilde{E}^{\ell}\left(k_{j}\right)$ of the DNS fields at $N=3$ (left) and $N=1$ (right).
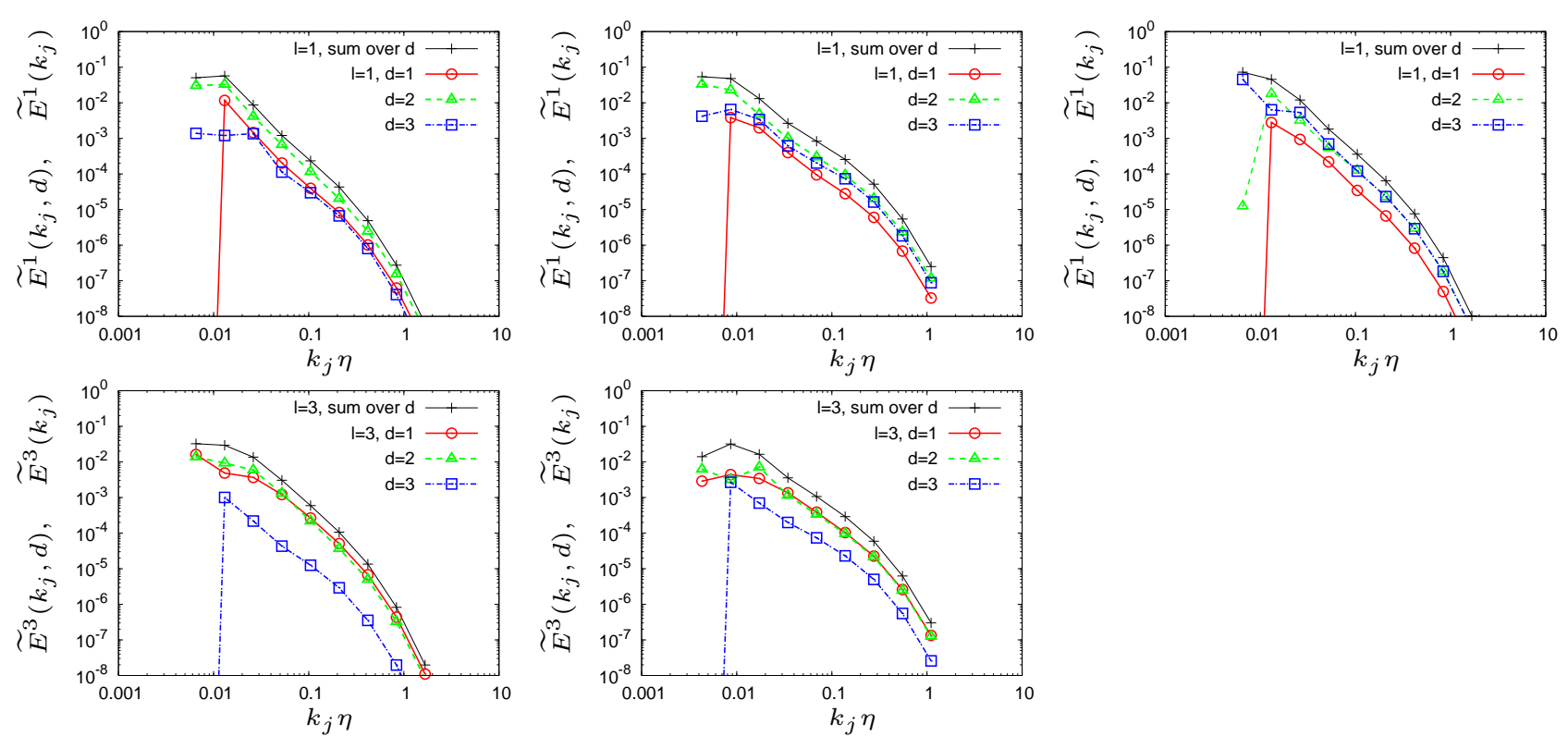

FIGURE 3. Directional wavelet energy spectra $\widetilde{E}^{\ell}\left(k_{j}, d\right)$ vs. $k_{j} \eta$ for the DNS field with $N=3$ (left), the DNS field with $N=1$ (middle) and the random field (right). For the isotropic random field only $\widetilde{E}^{1}\left(k_{j}, d\right)$ is plotted. The solid black lines with + show the corresponding wavelet mean energy spectra $\widetilde{E}^{\ell}\left(k_{j}\right)$.

velocity component parallel to $\boldsymbol{B}_{0}$, i.e., $\widetilde{E}^{3}\left(k_{j}\right)$, yields larger values than the spectra of the velocity components perpendicular to $\boldsymbol{B}_{0}$, i.e., $\widetilde{E}^{1}\left(k_{j}\right)$ and $\widetilde{E}^{2}\left(k_{j}\right)$, at each scale satisfying $k_{j} \eta \gtrsim 0.03(j=3,4, \cdots, 8)$. We also observe $\widetilde{E}^{1}\left(k_{j}\right) \sim \widetilde{E}^{2}\left(k_{j}\right)$. The predominance of $\widetilde{E}^{3}\left(k_{j}\right)$ over both $\widetilde{E}^{1}\left(k_{j}\right)$ and $\widetilde{E}^{2}\left(k_{j}\right)$ is consistent with the results of Burattini et al. [4], who examined Fourier spectra of each velocity component. For the field with $N=1$ (Figure 2, right), we observe that the wavelet mean energy spectra $\widetilde{E}^{\ell}\left(k_{j}\right)(\ell=1,2,3)$ agree well for $k_{j} \eta \gtrsim 0.03$. In the following, we consider only the statistics of the two velocity components, $u^{1}$ and $u^{3}$, because the considered system is axisymmetric with respect to the $x_{3}$-axis.

To examine the directional anisotropy of $u^{\ell}$ at each scale, we plot directional wavelet energy spectra $\widetilde{E}^{\ell}\left(k_{j}, d\right)$ for both velocity components in Figure 3 . For $N=3$ the largest energy contribution in $\widetilde{E}^{1}\left(k_{j}\right)$ is made by 


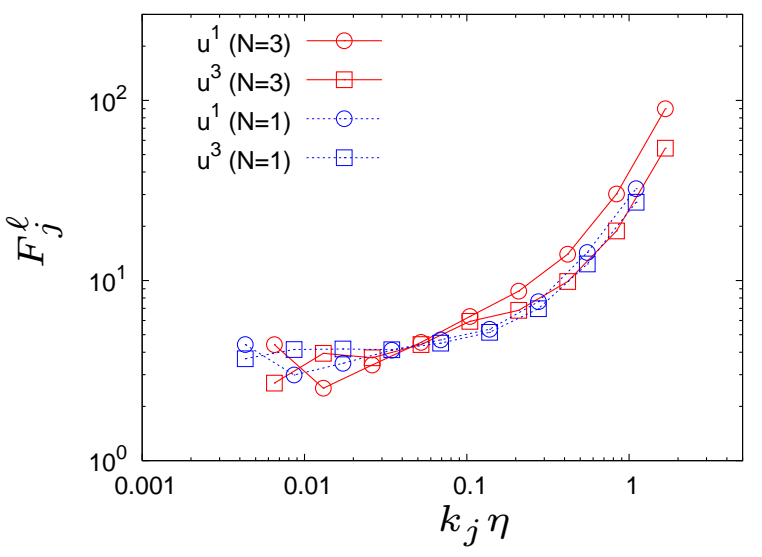

Figure 4. Scale-dependent flatness $F_{j}^{\ell}$ vs. $k_{j} \eta$ for the DNS fields with $N=1$ and $N=3$.

$\widetilde{E}^{1}\left(k_{j}, 2\right)$, followed by the remaining spectra, $\widetilde{E}^{1}\left(k_{j}, 1\right)$ and $\widetilde{E}^{1}\left(k_{j}, 3\right)$. Most of the energy of $\widetilde{E}^{3}\left(k_{j}\right)$ at $k_{j} \eta \gtrsim 0.03$ is retained by $\widetilde{E}^{3}\left(k_{j}, 1\right)$ and $\widetilde{E}^{3}\left(k_{j}, 2\right)$. The contribution of $\widetilde{E}^{3}\left(k_{j}, 3\right)$ to $\widetilde{E}^{3}\left(k_{j}\right)$ is almost negligible for $k_{j} \eta \gtrsim 0.03$.

The statistics for the field at $N=1$ confirm the weaker anisotropy compared to the case $N=3$, as anticipated from Figure 1. The spectra show that $\widetilde{E}^{1}\left(k_{j}, 2\right) \sim \widetilde{E}^{1}\left(k_{j}, 3\right)$ for $k_{j} \eta \gtrsim 0.03$, while the contribution of $\widetilde{E}^{1}\left(k_{j}, 1\right)$ is smaller.

We use the statistics of the isotropic random field as a reference to measure the anisotropy of the DNS fields. We see that $\widetilde{E}^{1}\left(k_{j}, 2\right)$ and $\widetilde{E}^{1}\left(k_{j}, 3\right)$ have almost the same contributions at $k_{j} \eta \gtrsim 0.03$, while the contribution of $\widetilde{E}^{1}\left(k_{j}, 1\right)$ is less pronounced compared to them. The reduced contribution of $\widetilde{E}^{1}\left(k_{j}, 1\right)$ compared to $\widetilde{E}^{1}\left(k_{j}, 2\right)$ and $\widetilde{E}^{1}\left(k_{j}, 3\right)$ is due to the divergence-free constraint of the flow, i.e., the longitudinal energy contribution of a given velocity component is reduced with respect to the transversal ones [10]. For the divergence-free random field we thus see the same trend in $\widetilde{E}^{\ell}\left(k_{j}, d\right)$, as that for the DNS field with $N=1$.

\subsection{Directional spatial intermittency}

The spatial intermittency of the DNS fields for $N=1$ and 3 is examined in Figure 4 by plotting the scaledependent flatness of $u^{\ell}, F_{j}^{\ell}$, vs. $k_{j} \eta$. For both DNS fields we can observe that $F_{j}^{\ell}(\ell=1,3)$ increases as scale decreases. We also find that the velocity component $u^{1}$ is more intermittent for $N=3$, than that for the field with $N=1$, for $k_{j} \eta \gtrsim 0.1$. In contrast, the flatness of the $u^{3}$ component only weakly depends on $N$.

To get further insight into the anisotropy of intermittency, we plot in Figure 5 the directional scale-dependent flatness $F_{j, d}^{\ell},(d=1,2,3)$ for the two DNS fields and the random field. For $N=3$ we find for both velocity components $\ell=1,3$ that $F_{j, 3}^{\ell}>F_{j, 1}^{\ell}$ and $F_{j, 3}^{\ell}>F_{j, 2}^{\ell}$ at each scale satisfying $k_{j} \eta \gtrsim 0.3$. For both velocity components $\ell=1,3$ and all three directions $d=1,2,3$ the flatness values $F_{j, d}^{\ell}$ are larger for $N=3$ than for $N=1$. In particular the flatness in the $z$-direction, $F_{j, 3}^{\ell}$, is significantly larger for the case $N=3$ than for $N=1$. For the field with $N=1, F_{j, d}^{\ell}$ is larger than $F_{j, \ell}^{\ell}$ for $d \neq \ell$. This predominance of $F_{j, d}^{\ell}$ over $F_{j, \ell}^{\ell}$ for $d \neq \ell$ is consistent with the two cases of isotropic and rotating incompressible turbulence studied in [10]. As anticipated the random field has values close to three for $k_{j} \eta \gtrsim 0.02$, which is characteristic for Gaussian noise (Figure 5, right).

These results on the flatness $F_{j, d}^{\ell}$ show that the imposed magnetic field $\boldsymbol{B}_{0}$ plays a significant role on the amplification of small-scale intermittency, especially the intermittency in the $x_{3}$-direction, which is parallel to $\boldsymbol{B}_{0}$. This is in contrast to the case of incompressible rotating turbulence, whose flow structures are aligned parallel with the rotational axis. The rotational effect does not play a major role on the amplification of small-scale intermittency [10]. 

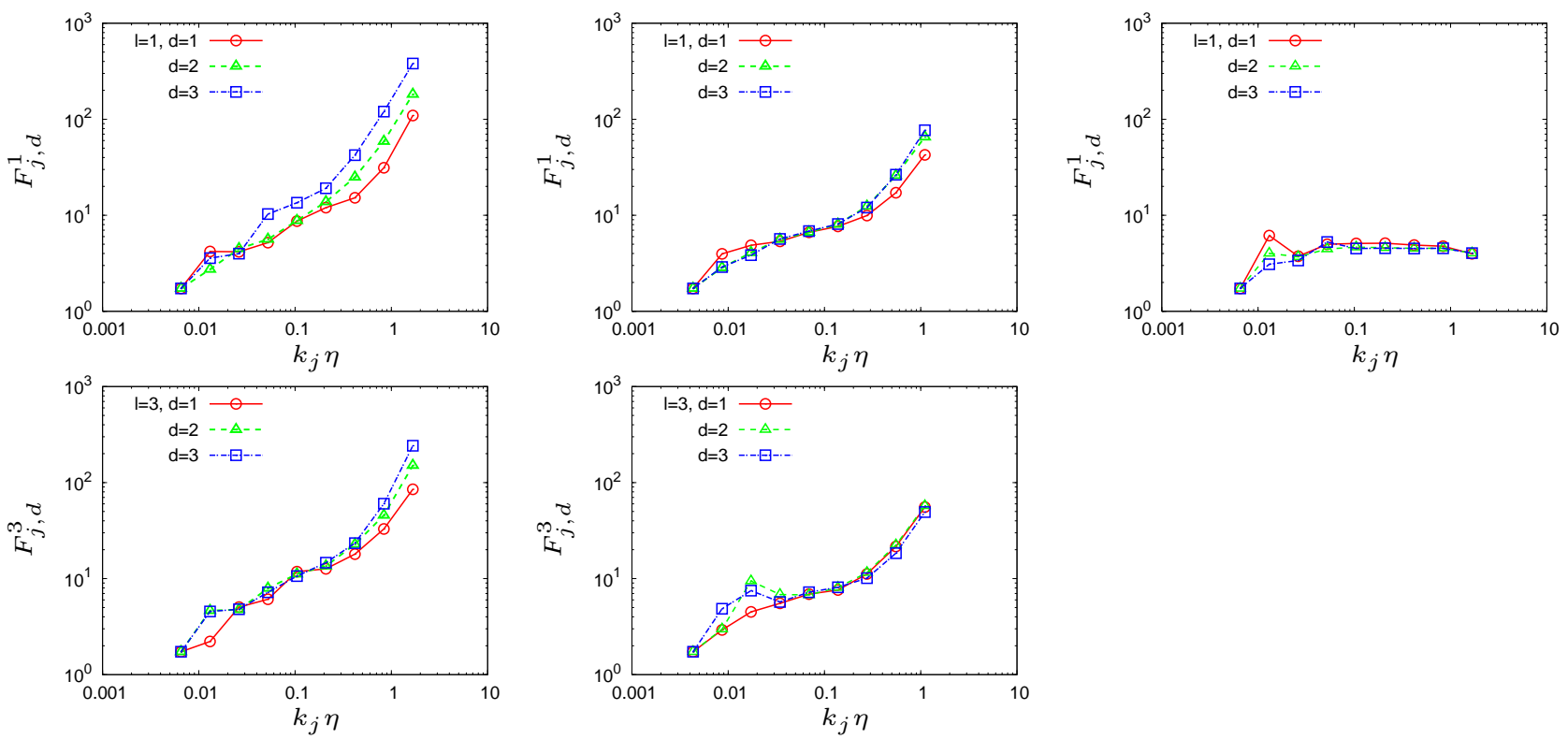

Figure 5. Directional scale-dependent flatness for the DNS field with $N=3$ (left), the DNS field with $N=1$ (middle), and the isotropic Gaussian random field (right). For the random field only $F_{j, d}^{1}$ is plotted, as $F_{j, d}^{3}$ yields similar values.

\section{Conclusion}

The anisotropy and intermittency of homogeneous incompressible quasi-static MHD turbulence with an imposed magnetic field $\boldsymbol{B}_{0}$ have been examined using three-dimensional orthonormal wavelet decomposition. Wavelet analysis is an efficient tool to examine directional scale-dependent statistics, since it is based on welllocalized functions in space, scale and direction. The wavelet representation detects and allows to quantify the flow anisotropy and intermittency which are directly reflected in the coefficients. In the presented analyzes, we focused on directions parallel and perpendicular to $\boldsymbol{B}_{0}$. We analyzed two DNS data sets computed with $512^{3}$ grid points of turbulent MHD fields with two different interaction parameters, $N=1$ and 3 . The results were compared with those obtained for an isotropic divergence-free Gaussian random field with the same energy spectrum as that of the turbulent field at $N=3$.

The directional wavelet energy spectra allowed to characterize the anisotropy of the turbulent fields at each scale. The energy for the field at $N=3$ was shown to be dominated by the perpendicular direction at each scale. In contrast, the directional dependence of energy for the field with $N=1$ was shown to be qualitatively close to that of the isotropic random field.

The results confirmed that the imposed magnetic field $\boldsymbol{B}_{0}$ plays a significant role not only for the anisotropybut also for the amplification of small-scale intermittency, especially in the direction parallel to $\boldsymbol{B}_{0}$. In contrast, the random field did not show any intermittent behavior, as expected.

The present results provide useful information for the improvement of wavelet-based turbulence models for anisotropic turbulence. A wavelet-based turbulence model, the Coherent Vorticity Simulation (CVS), which is based on the deterministic computation of the coherent vorticity field using an adaptive wavelet basis, was proposed in $[17,18]$, for a review we refer to [15]. The coherent vorticity field is obtained by filtering the wavelet coefficients, which thus exploits the intermittency of turbulence and yields a sparse representation. Examples of CVS can be found in [17-20] for two-dimensional turbulent flows and in [21] for three-dimensional turbulent 
mixing layers. For highly anisotropic turbulence, the wavelet-based simulation method is expected to become more efficient, if one takes into account the anisotropy and intermittency of the turbulence.

\section{ACKNOWLEDGMENTS}

The computations were carried out on the Earth Simulator and the FX1 system at the Information Technology Center of Nagoya University. K.Y. and N.O. are grateful for Grant-in-Aids for Young Scientists (B) 22740255 and 22760063, respectively, from the Ministry of Education, Culture, Sports, Science and Technology. M.F. and K.S. thankfully acknowledge financial support from the PEPS program of INSMI-CNRS and also thank theAssociation CEA-EURATOM and the FRF2S (French Research Federation for FusionStudies) for supporting their work within the framework of the EFDA (European FusionDevelopment Agreement) under contract V.3258.001. We acknowledge the CIRM, Luminy, for hospitality during the 2010 CEMRACS summer program on "Numerical modelling of fusion".

\section{REFERENCES}

[1] P. A. Davidson, An Introduction to Magnetohydrodynamics, Cambridge University Press, (2001).

[2] B. Knaepen, S. Kassinos and D. Carati, Magnetohydrodynamic turbulence at moderate magnetic Reynolds number, J. Fluid Mech., 513, (2004), 199-220.

[3] B. Knaepen and R. Moreau, Magnetohydrodynamic Turbulence at Low Magnetic Reynolds Number, Annu. Rev. Fluid Mech. 40, (2008), 25-45.

[4] P. Burattini, M. Kinet, D. Carati and B. Knaepen, Anisotropy of velocity spectra in quasistatic magnetohydrodynamic turbulence, Phys. Fluids, 20, (2008), 065110.

[5] A. Vorobev, O. Zikanov, P. A. Davidson and B. Knaepen, Anisotropy of MHD turbulence at low magnetic Reynolds number, Phys. Fluids, 17, (2005), 125105.

[6] T. IshidA, AND Y. KANEDA Small-scale anisotropy in magnetohydrodynamic turbulence under a strong uniform magnetic field, Phys. Fluids, 19, (2007), 075104.

[7] S. Mallat, A wavelet tour of signal processing, Third Edition: The Sparse Way, Academic Press (2010).

[8] C. Meneveau, Analysis of turbulence in the orthonormal wavelet representation, J. Fluid Mech., 232, (1991), 469-520.

[9] M. FARGE, Wavelet transforms and their applications to turbulence, Annu. Rev. Fluid Mech. 24, (1992), 395-457.

[10] W. Bos, L. Liechtenstein And K. Schneider, Small scale intermittency in anisotropic turbulence, Phys. Rev. E, 76, (2007), 046310.

[11] K. Yoshimatsu, N. Okamoto, K. Schneider, Y. Kaneda and M. Farge, Intermittency and scale-dependent statistics in fully developed turbulence, Phys. Rev. E, 79, (2009), 026303.

[12] F. G. Jacobitz, K. Schneider, W. J. Bos And M. Farge, On the structure and dynamics of sheared and rotating turbulence: Anisotropy properies and geometrical scale-dependent statistics, Phys. Fluids, 22, (2010), 085101.

[13] M. Farge, G. Pellegrino And K. Schneider, Coherent vortex extraction in $3 d$ turbulent flows using orthogonal wavelets, Phys. Rev. Lett. 87, (2001), 45011-45014.

[14] K. Yoshimatsu, Y. Kondo, K. Schneider, N. Okamoto, H. Hagiwara and M. Farge, Wavelet-based coherent vorticity sheet and current sheet extraction from three-dimensional homogeneous magnetohydrodynamic turbulence, Phys. Plasmas, 16, (2009), 082306.

[15] K. Schneider, And O. VAsilyev, Wavelet Methods in Computational Fluid Dynamics, Annu. Rev. Fluid Mech., 42, (2010), 473-503.

[16] K. YoshidA, AND T. ARIMITSU, Inertial-subrange structures of isotropic incompressible magnetohydrodynamic turbulence in the Lagrangian renormalized approximation, Phys. Fluids, 19, (2007), 045106.

[17] M. Farge, K. Schneider and N. Kevlahan, Non-Gaussianity and coherent vortex simulation for two-dimensional turbulence using an adaptive orthonormal wavelet basis, Phys. Fluids, 11, (1999), 2187-2201.

[18] M. FARge, AND K. Schneider, Coherent Vortex Simulation (CVS), a semi-deterministic turbulence model using wavelets, Flow, Turbul. Combust. 66, (2001), 393-426.

[19] J. FröHlich ANd K. SChneIder, Computation of decaying turbulence in an adaptive wavelet basis, Physica D, 134, (1999), $337-361$.

[20] K. Schneider, M. Farge, A. Azzalini and J. Ziuber, Coherent vortex extraction and simulation of 2d isotropic turbulence, J. Turbul. 7, No. 44, (2006), 1-24.

[21] K. Schneider, M. Farge, G. Pellegrino and M. Rogers, Coherent vortex simulation of $3 D$ turbulent mixing layers using orthogonal wavelets, J. Fluid Mech., 534, (2005), 39-66. 